\title{
RB217, RB218 and RB219 antibodies recognize Protoporphyrin IX by ELISA
}

\author{
Philippe Hammel, Norbert Lange \\ School of Pharmaceutical Sciences, University of Geneva, Rue Michel Servet 1, 1211, Geneva, Switzerland \\ Institute of Pharmaceutical Sciences of Western Switzerland, University of Geneva, Rue Michel Servet 1, 1211, Geneva, Switzerland
}

\begin{abstract}
The recombinant antibodies RB217, RB218 and RB219 detect protoporphyrin IX by ELISA.
\end{abstract}

\section{Introduction}

Protoporphyrin IX (PpIX, ChEBI 15430), a heterocyclic organic compound with four pyrrole rings, is the biosynthetic precursor of hemes and chlorophylls. Heme (ChEBI 30413) consists of PpIX with a Fe(II) center (Sitte and Senge, 2020). PpIX is also used as photosensitizer in photodynamic therapy (PDT) (Sansaloni-Pastor et al., 2019). Here we describe the ability of three recombinant antibodies (RB217, RB218 and RB219) to detect PpIX by ELISA.

\section{Materials \& Methods}

Antibodies: ABCD_RB217, ABCD_RB218 and ABCD_RB219 antibodies (ABCD nomenclature, https://web.expasy.org/abcd/) were produced by the Geneva Antibody Facility (https://www.unige.ch/ medecine/antibodies) as mini-antibodies with the antigenbinding scFv portion fused to a rabbit Fc. HEK293T cells (growing in DMEM GlutaMAXTM (Gibco 31966) supplemented with 8\% Fetal Bovine Serum (Gibco 10270)) were transiently transfected with the vector coding for the scFv-Fc of each antibody. Supernatants $(\sim 5$ $\mathrm{mg} / \mathrm{L}$ ) were collected after 3 days.

Antigen: The antibodies were raised against biotinylated protoporphyrin IX. As a control, biotinylated heme (PpIX with $\mathrm{Fe}^{2+}$ ) and an irrelevant biotinylated peptide (LWSFTPSKCSGPYGE) were used.

Protocol: The whole procedure was carried out at room temperature. Biotinylated PpIX and heme at $4 \mu \mathrm{g} / \mathrm{ml}$ were immobilized on streptavidin-coated ELISA plates (Pierce 15124) for $30 \mathrm{~min}$. Each well was rinsed three times with $100 \mu 1$ of washing buffer (PBS $+0.5 \%(\mathrm{w} / \mathrm{v}) \mathrm{BSA}+0.05 \%$ (w/v) Tween20), then incubated for 1 hour with $50 \mu \mathrm{l}$ of antibody-containing supernatant diluted in washing buffer. After rinsing 3 times (100 $\mu 1$ washing buffer), wells were incubated with horseradish peroxidase-coupled goat anti-rabbit IgG (Sigma A8275, dilution 1:1000, $50 \mu \mathrm{l}$ per well) for $30 \mathrm{~min}$. After 3 rinses, Tetramethylbenzidine (TMB) substrate (Sigma T5569) was added (50 $\mu$ per well). The reaction was stopped by the addition of $25 \mu \mathrm{l}$ of $2 \mathrm{M} \mathrm{H}_{2} \mathrm{SO}_{4}$. The absorbance (OD) was measured at 450 $\mathrm{nm}$, and the absorbance at $570 \mathrm{~nm}$ was subtracted.

\section{Results}

Antibodies RB217, RB218 and RB219 bound in a concentration-dependent manner to protoporphyrin IX (PP) (Fig. 1). Antibodies RB218 and RB219 also bound to heme, but not RB217 (Fig. 1). The antibodies did not bind to an irrelevant control peptide (Fig. 1).
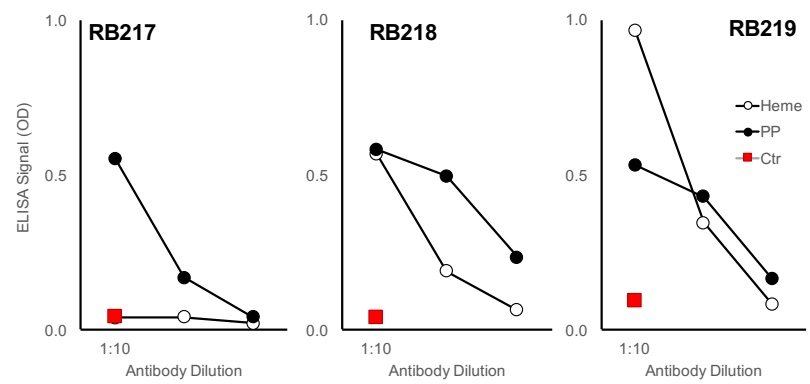

Fig. 1. Binding of RB antibodies to Protoporphyrin IX (PP), Heme and a control peptide (Ctr, values shown only for the 1:10 antibody dilution), as detected by ELISA.

\section{References}

Sansaloni-Pastor S, Bouilloux J, Lange N. The Dark Side: Photosensitizer Prodrugs. Pharmaceuticals (Basel). 2019; 12(4):148. PMID: 31590223.

Sitte E, Senge MO. The red color of life transformed synthetic advances and emerging applications of protoporphyrin IX in chemical biology. European J Org Chem. 2020; 2020:3171-3191. PMID:32612451

\section{Conflict of interest}

The authors declare no conflict of interest. 